\title{
Möbius Transformations of the Circle Form a Maximal Convergence Group
}

\author{
Ara Basmajian and Mahmoud Zeinalian
}

\begin{abstract}
We investigate the relationship between quasisymmetric and convergence groups acting on the circle. We show that the Möbius transformations of the circle form a maximal convergence group. This completes the characterization of the Möbius group as a maximal convergence group acting on the sphere. Previously, Gehring and Martin had shown the maximality of the Möbius group on spheres of dimension greater than one. Maximality of the isometry (conformal) group of the hyperbolic plane as a uniform quasiisometry group, uniformly quasiconformal group, and as a convergence group in which each element is topologically conjugate to an isometry may be viewed as consequences.
\end{abstract}

\section{Introduction}

The isometries of real hyperbolic space of dimension two or higher induce conformal diffeomorphisms on its ideal boundary. In fact, if the dimension is strictly greater than two, then all conformal diffeomorphisms will arise in this way. In contrast, in dimension two, conformality on the boundary is a trivial condition. For instance, every diffeomorphism of a Riemannian circle preserves the conformal class of the metric. It is for this reason that the study of the group of Möbius transformations of the circle differs from its higher dimensional cousins. In the paper $[\mathbf{G e}-\mathbf{M}]$, Gehring and Martin show that the Möbius group is a maximal convergence group acting on the boundary of real hyperbolic space of dimension greater than two. This result was extended (see $[\mathbf{B}-\mathbf{Z}]$ ) to the action of the isometry group of a rank one symmetric space of noncompact type except the hyperbolic plane.

In this note, we complete the characterization of the Möbius group as a maximal convergence group by considering the remaining case of the hyperbolic plane; namely, the group of Möbius transformations of the circle acts as a maximal convergence group (Theorem 3.3). Other maximality statements, such as the maximality of the isometry (conformal) group of the hyperbolic plane as a uniform quasiisometry group and a uniformly quasiconformal group (see Corollary 4.2 and the discussion at the end of that section) may be regarded as consequences of Theorem 3.3 See Gr-P for further discussion on quasi-isometry groups. Another implication is the maximality of the isometry group of the hyperbolic plane as a

2000 Mathematics Subject Classification. Primary 30C62, Secondary 30F40.

Key words and phrases. convergence group, quasisymmetric, Möbius. 
convergence group in which each element is topologically conjugate to an isometry (Corollary 4.3).

Let $X$ be a compact topological space. A family $\mathcal{F}$ of orientation preserving homeomorphisms of $X$ is said to have the convergence property if each infinite sequence $\left\{f_{n}\right\}$ of $\mathcal{F}$ contains a subsequence which,

C1: converges uniformly to a homeomorphism of $X$, or

C2: has the attractor-repeller property, that is, there exists a point $a \in X$, the attractor, and a point $r \in X$, the repeller, so that the $\left\{f_{n}\right\}$ converge to the constant function $a$, uniformly outside of any open neighborhood of $r$. Note that $a$ may equal $r$.

We remark that the convergence groups considered in this paper are comprised only of orientation preserving homeomorphisms. We could equally as well include orientation reversing homeomorphisms, in which case the theorems in this paper have obvious modifications that are left to the reader. Hence, whenever homeomorphism is mentioned in this paper it is assumed to be orientation preserving.

\section{Elementary facts about quasisymmetric mappings}

In this section, we assemble some elementary facts which will be needed later in the paper. For the basics on quasisymmetric and quasiconformal maps, we refer to the following papers and books: $\underline{\mathbf{A}}, \mathbf{D}-\mathbf{E}, \mathbf{G a - L}, \underline{\mathbf{H}}, \mathbf{\mathbf { L }}$, and $\underline{\mathbf{V}}$. For Möbius groups and hyperbolic geometry, the reader may consult $[\mathbf{B e}$ or $[\mathbf{M}]$.

Let $\mathbb{H}$ denote the upper half plane, endowed with the hyperbolic metric, and $\widehat{\mathbb{R}}=\mathbb{R} \cup\{\infty\}$ denote its ideal boundary. $\widehat{\mathbb{R}}$ can be identified with the unit circle $S^{1}$. The group $\operatorname{PSL}(2, \mathbb{R})=\left\{z \mapsto \frac{a z+b}{c z+d}: a, b, c, d \in \mathbb{R}, a d-b c=1\right\}$ is the full group of orientation preserving isometries of $\mathbb{H}$. This group is also the full group of conformal homeomorphisms of $\mathbb{H}$. Let $\mathrm{Möb}^{+}(\widehat{\mathbb{R}})$ denote the group of homeomorphisms of $\widehat{\mathbb{R}}$ which are induced by the isometries of $\mathbb{H}$. Note that $\operatorname{PSL}(2, \mathbb{R})$ and $\mathrm{Möb}^{+}(\widehat{\mathbb{R}})$ are isomorphic groups which act on different spaces.

When dealing with mappings of the circle we will need to normalize by post composition using an element of $\mathrm{Möb}^{+}(\widehat{\mathbb{R}})$ so that infinity is a fixed point of the map. Observe that an element of the stabilizer of $\infty$ in $\operatorname{Möb}^{+}(\widehat{\mathbb{R}})$ is a linear map, $x \mapsto m x+b$, where $m>0$ and $b \in \mathbb{R}$. Hence given a symmetric configuration of point triples, $\{x-t, x, x+t\}$, its image remains a symmetric configuration.

Definition 2.1. Let $f: \widehat{\mathbb{R}} \rightarrow \widehat{\mathbb{R}}$ be an orientation preserving homeomorphism and $k>0$. The homeomorphism $f$ is called $k$-quasisymmetric if after normalizing so that it fixes infinity, $f$ satisfies,

$$
\frac{1}{k} \leq \frac{f(x+t)-f(x)}{f(x)-f(x-t)} \leq k
$$

for all $x \in \mathbb{R}$ and all $t>0$. In other words, the image of equal length juxtaposed intervals has uniform bounded length ratio.

Given the observation preceding Definition 2.1] it is easy to see that the condition of being $k$-quasisymmetric is independent of the normalizing Möbius transformation, the elements of $\mathrm{Möb}^{+}(\widehat{\mathbb{R}})$ are 1-quasisymmetric, and that post or precomposition by linear maps does not change the quasisymmetric constant.

In the sequel, we will need the fact that $\mathrm{Möb}^{+}(\widehat{\mathbb{R}})$ is the full group of 1quasisymmetric homeomorphisms. To see this, using the triple transitivity of 
$\mathrm{Möb}^{+}(\widehat{\mathbb{R}})$, it is enough to show that a 1-quasisymmetric mapping $f$ which fixes 0,1 , and $\infty$ is the identity. Now, the triple $\left\{0, \frac{1}{2}, 1\right\}$ must be taken to a symmetric triple, hence $f$ also fixes $\frac{1}{2}$. Similar considerations allow us to conclude that all rationals of the form $\frac{n}{2^{k}}$ are fixed. Finally, using continuity, $f$ must fix every real number and thus is the identity homeomorphism.

The following proposition is a classical result. See Ahlfors $[\mathbf{A}$ or Lehto $\mathbf{L}$ for reference.

Proposition 2.2. Let $f: \widehat{\mathbb{R}} \rightarrow \widehat{\mathbb{R}}$ be an increasing homeomorphism with $f(\infty)=\infty$. Assume that

$$
\frac{1}{k} \leq \frac{f(x+t)-f(x)}{f(x)-f(x-t)} \leq k
$$

for all $x$ and all $t>0$. Then there exists a $K(k)$-quasiconformal homeomorphism of $\mathbb{H}$ which extends $f$. The number $K(k)$ depends only on $k$.

\section{Maximality of $\mathrm{Möb}^{+}(\widehat{\mathbb{R}})$}

A family $\mathcal{F}$ is said to be uniformly quasisymmetric (quasiconformal) if all the maps in $\mathcal{F}$ are $k$-quasisymmetric ( $K$-quasiconformal) for some $k$ (for some $K$ ).

Proposition 3.1. A uniformly quasisymmetric family $\mathcal{F}$ of homeomorphisms of $\widehat{\mathbb{R}}$ is a convergence family. In particular, $M \ddot{b} b^{+}(\widehat{\mathbb{R}})$ is a convergence group.

Proof. Using Proposition 2.2 there exists a number $K$ such that every element of this family can be extended to a $K$-quasiconformal mapping of $\mathbb{H}$. Using the fact that a sequence of distinct $K$-quasiconformal mappings of $\mathbb{H}$ has a subsequence which either converges to a $K$-quasiconformal map or has the attractorrepeller property with attractor and repeller on the boundary (see $\mathbf{V}$, Corollaries 19.3 and 37.4, or extend each map in $\mathcal{F}$ to $S^{2}$ by reflection and use the results of [Ge-M]), we may conclude it acts as a convergence family on $S^{1}$.

Proposition 3.2. Let $\mathcal{F}$ be a family of homeomorphisms of $\widehat{\mathbb{R}}$ which is closed under post and precomposition by elements of $\operatorname{Möb}^{+}(\widehat{\mathbb{R}})$. Then $\mathcal{F}$ has the convergence property if and only if $\mathcal{F}$ is a uniformly quasisymmetric family.

Proof. Suppose $\mathcal{F}$ has the convergence property. Let

$$
\mathcal{F}^{\prime}=\{f \in \mathcal{F}: f(0)=0, f(1)=1 \text {, and } f(\infty)=\infty\} .
$$

Since $\mathcal{F}^{\prime}$ has the convergence property, it must be that there are negative constants $M$ and $m$ so that, $m<f(-1)<M<0$, for all $f \in \mathcal{F}^{\prime}$. Any element of $\mathcal{F}$ can be post composed by an element of Möb $(\widehat{\mathbb{R}})$ to yield an element of $\mathcal{F}^{\prime}$. Since any triple $\{x-t, x, x+t\}$ in $\mathbb{R}$ can be moved by Euclidean translation and dilation to $\{-1,0,1\}$, we may conclude that the elements of $\mathcal{F}$ form a uniformly quasisymmetric family. The converse follows from Proposition 3.1

TheOREM 3.3. Möb $(\widehat{\mathbb{R}})$ acts on $\widehat{\mathbb{R}}$ as a maximal convergence group. That is, there is no convergence group that properly contains $\mathrm{Möb}^{+}(\widehat{\mathbb{R}})$.

Proof. The fact that $\mathrm{Möb}^{+}(\widehat{\mathbb{R}})$ is a convergence group follows from Proposition 3.1 Next let $G$ be a convergence group acting on $\widehat{\mathbb{R}}$ containing $\operatorname{Möb}^{+}(\widehat{\mathbb{R}})$. 
Using Proposition 3.2 we know that the action of $G$ is as a uniformly quasisymmetric group. On the other hand, suppose there exists an element $g \in G$ not contained in $\mathrm{Möb}^{+}(\widehat{\mathbb{R}})$. This means that after normalizing $g$, so that it fixes $\infty$, there must be three symmetrically spaced points in $\mathbb{R}$ where the quasisymmetric constant is not 1. Post and precomposing by Euclidean translations and dilations, we may assume that the three points are $\{-1,0,1\}$ and that $g$ fixes 0 and 1 . Since Euclidean translation and dilation do not effect the quasisymmetric constant for a triple, it must be that $g$ does not fix -1 . By possibly replacing $g$ with $g^{-1}$, we may assume that $g$ takes -1 into the interval $(-1,0)$. Clearly $g^{n}(-1)$ is an increasing sequence of negative numbers and hence has a limit $y$ which is necessarily a fixed point of $g$. Since $\langle g\rangle$ is a convergence group, $y$ is strictly less than 0 . Next consider the triple of points $\{-1, y, 0\}$. The length ratio of the juxtaposed intervals $[-1, y]$ and $[y, 0]$ is,

$$
\frac{|0-y|}{|y-(-1)|}=\frac{|y|}{|y+1|}
$$

The length ratio of image intervals under the iterates of $g$ are

$$
\frac{\left|g^{n}(0)-g^{n}(y)\right|}{\left|g^{n}(y)-g^{n}(-1)\right|}=\frac{|y|}{\left|y-g^{n}(-1)\right|}
$$

which goes to $\infty$, as $n \rightarrow \infty$. This contradicts the fact that $G$ is uniformly quasisymmetric. Hence, it must be that the quasisymmetric constant for $g$ is 1 , and thus $g \in \mathrm{Möb}^{+}(\widehat{\mathbb{R}})$.

\section{Maximality of $\operatorname{PSL}(2, \mathbb{R})$}

An immediate corollary of Theorem 3.3 is,

Corollary 4.1. The Möbius group, Möb $b^{+}(\widehat{\mathbb{R}})$, is a maximal uniformly quasisymmetric group.

A homeomorphism $f: \mathbb{H} \rightarrow \mathbb{H}$ is said to be a quasi-isometry if there exist positive constants $A$ and $B$ so that

$$
A^{-1} d\left(x_{1}, x_{2}\right)-B \leq d\left(f\left(x_{1}\right), f\left(x_{2}\right)\right) \leq A d\left(x_{1}, x_{2}\right)+B
$$

for all $x_{1}, x_{2} \in \mathbb{H}$. The constant $A$ is referred to as the Lipschitz constant of the quasi-isometry. For a general reference on quasi-isometries, we refer the reader to $\mathbf{G r}-\mathbf{P}$. It is well known that a quasi-isometry continuously extends to the boundary and that the induced map on the boundary is a quasisymmetric homeomorphism. One defines an equivalence relation on quasi-isometries by declaring two to be equivalent if they induce the same homeomorphism on the boundary. Let $\mathrm{QI}(\mathbb{H})$ denote the group of equivalence classes of quasi-isometries of $\mathbb{H}$. Since the natural map from $\operatorname{PSL}(2, \mathbb{R})$ into $\mathrm{QI}(\mathbb{H})$ is injective, we will continue to denote its image with the same notation. A family $\mathcal{F} \subset \mathrm{QI}(\mathbb{H})$ is said to be a uniformly quasi-isometric family if each equivalence class has Lipschitz constant less than a uniform bound. The following is a simple consequence of Theorem 3.3 observed in Gromov and Pansu (see Gr-P).

COROLLARY 4.2. Let $G \leq Q I(\mathbb{H})$ be a uniform quasi-isometry group acting on the hyperbolic plane $\mathbb{H}$. If $P S L(2, \mathbb{R}) \leq G$, then $G=\operatorname{PSL}(2, \mathbb{R})$. 
Proof. A quasi-isometry $f$ of $\mathbb{H}$ extends to a homeomorphism of $\mathbb{H} \cup \widehat{\mathbb{R}}$. Moreover, the induced mapping $\left.f\right|_{\widehat{\mathbb{R}}}$ on $\widehat{\mathbb{R}}$ is a quasisymmetric mapping where the quasisymmetric constant depends only on the Lipschitz constant of the quasi-isometry. Consider the homomorphism $\phi: G \rightarrow \operatorname{Homeo}(\widehat{\mathbb{R}})$, given by $\left.[f] \mapsto f\right|_{\widehat{\mathbb{R}}}$. Note that Image $(\phi)$ is a uniformly quasisymmetric group which contains $\mathrm{Möb}^{+}(\widehat{\mathbb{R}})$. By Proposition 3.1. Image $(\phi)$ is a convergence group. Since $\mathrm{Möb}^{+}(\widehat{\mathbb{R}})$ is a maximal convergence group (Theorem [3.3), Image $(\phi)$ equals $\mathrm{Möb}^{+}(\widehat{\mathbb{R}})$. Injectivity of $\phi$ is a tautology. Since Image $(\phi)=\phi(\operatorname{PSL}(2, \mathbb{R}))=\operatorname{Möb}^{+}(\widehat{\mathbb{R}})$, we conclude that $G=\operatorname{PSL}(2, \mathbb{R})$.

As in the proof above, it is easy to see that a convergence group acting on $\mathbb{H} \cup \widehat{\mathbb{R}}$ which contains $\operatorname{PSL}(2, \mathbb{R})$ induces the action of $\mathrm{Möb}^{+}(\widehat{\mathbb{R}})$ on the boundary, $\widehat{\mathbb{R}}$. Furthermore, if each element of this convergence group is topologically conjugate to an element of $\operatorname{PSL}(2, \mathbb{R})$, then the induced action has trivial kernel. Hence the natural homomorphism given by restriction of the convergence group to the boundary is in fact an isomorphism onto $\mathrm{Möb}^{+}(\widehat{\mathbb{R}})$. Since the image of $\operatorname{PSL}(2, \mathbb{R})$ is $\operatorname{Möb}^{+}(\widehat{\mathbb{R}})$, it must be that the convergence group equals $\operatorname{PSL}(2, \mathbb{R})$. We have proven,

COROLLARY 4.3. Let $G$ be a convergence group acting on $\mathbb{H} \cup \widehat{\mathbb{R}}$. Suppose that every element of $G$ is topologically conjugate to an element of $\operatorname{PSL}(2, \mathbb{R})$. If $P S L(2, \mathbb{R}) \leq G$, then $G=P S L(2, \mathbb{R})$. The conjugating homeomorphism need not be the same for all elements of $G$.

The reader should compare the above corollary to the fact that $\operatorname{PSL}(2, \mathbb{R})$ is a maximal uniformly quasiconformal group acting on $\mathbb{H}$. That is, if $G$ is a uniformly quasiconformal group containing $\operatorname{PSL}(2, \mathbb{R})$, then $G=\operatorname{PSL}(2, \mathbb{R})$. This fact follows from the results of $[\underline{\mathbf{S}}$ and $[\mathbf{T}$.

\section{References}

[A] L. V. Ahlfors, Lectures on Quasiconformal Mappings, Van Nostrand-Reinhold, Princeton, New Jersey, (1966).

[Be] Alan F. Beardon, The geometry of discrete groups, Graduate Texts in Mathematics, 91. Springer-Verlag, New York, 1983. xii+337 pp.

[B-Z] A. Basmajian, M. Zeinalian, Maximal convergence groups and rank one symmetric spaces, preprint.

[D-E] A. Douady, C. J. Earle, Conformally natural extension of homeomorphisms of the circle, Acta Math. 157 no. 1-2 (1986), 23-48.

[Ga-L] F. P. Gardiner, N. Lakic, Quasiconformal Teichmuller Theory, American Mathematical Society, Mathematical Surveys and Monographs, 76 (2000).

[Ge-M] F. W. Gehring, G. J. Martin, Discrete quasiconformal groups I, Proc. London Math. Soc. (3), Vol 55, (1987) 331-358.

[Gr-P] M. Gromov, P. Pansu, Rigidity of lattices: An introduction, Geometric Topology: Recent developments, Lecture Notes in Math. 1504, Springer, Berlin (1991), 39-137.

[H] A. Hinkkanen, Uniformly quasisymmetric groups. Proc. London Math. Soc. (3) 51 (1985), no. $2,318-338$

[L] O. Lehto, Univalent Functions and Teichmülar Spaces, Springer-Verlag, (1987).

[M] B. Maskit, Kleinian groups, Grundlehren der Mathematischen Wissenschaften [Fundamental Principles of Mathematical Sciences], 287. Springer-Verlag, Berlin, 1988. xiv +326 pp.

[S] D. Sullivan, On the ergodic theory at infinity of an arbitrary discrete group of hyperbolic motions, Riemann surfaces and related topics: Proceedings of the 1978 Stony Brook Conference (1978), 465-496; Ann. of Math. Stud., 97, Princeton Univ. Press (1981). 
[T] P. Tukia, On two-dimensional quasiconformal groups, Ann. Acad. Sci. Fenn. Ser. A I Math. 5 no. 1 (1980), 73-78.

[V] J. Vaisala, Lectures on n-dimensional quasiconformal mappings, Lecture Notes in Math. 229, Springer-Verlag, Berlin-New York (1971).

Ara Basmajian, Department of Mathematics, University of Oklahoma, Norman, OK 73019, abasmajian@ou.edu

Mahmoud Zeinalian, Department of Mathematics, C.W.Post Campus, Long Island University, 720 Northern Boulevard, Brookville, NY 11548, mzeinalian@liu.edu

Department of Mathematics, University of Oklahoma, Norman, Oklahoma 73019

E-mail address: abasmajian@ou.edu

Department of Mathematics, C.W.Post College of Long Island University, 720 Northern Boulevard, Brookville, New York 11548

E-mail address: mzeinalian@liu.edu 